Annuaire suisse de politique de développement

6 | 1987

Annuaire Suisse - Tiers Monde 1986

\title{
Pour un commerce équitable avec le Tiers Monde
}

\section{Andràs November}

\section{OpenEdition}

\section{Journals}

Édition électronique

URL : http://journals.openedition.org/aspd/1234

DOI : $10.4000 /$ aspd. 1234

ISSN : 1663-9669

\section{Éditeur}

Institut de hautes études internationales et du développement

\section{Édition imprimée}

Date de publication : 1 janvier 1987

Pagination : 161-176

ISSN : 1660-5934

\section{Référence électronique}

Andràs November, "Pour un commerce équitable avec le Tiers Monde », Annuaire suisse de politique de développement [En ligne], 6| 1987, mis en ligne le 06 mars 2013, consulté le 10 décembre 2020. URL: http://journals.openedition.org/aspd/1234 ; DOI : https://doi.org/10.4000/aspd.1234 


\title{
POUR UN COMMERCE EQUITABLE AVEC LE TIERS MONDE
}

\author{
Andràs November
}

Le 16 janvier 1986, sept organisations d'entraide suisses ont lancé un appel intitulé : «Pour un commerce équitable avec le Tiers Monde» (1). II s'adresse à la fois aux milieux économiques et aux autorités politiques. Le but visé par l'opération - au-delà de la collecte de signatures pour en faire une pétition - est d'attirer l'attention du consommateur suisse sur la situation sociale des producteurs du Tiers Monde. En même temps, elle veut le rendre conscient qu'au moment de l'achat dans un magasin, son choix des produits a des conséquences sociales qui touchent les pays en voie de développement (PVD). En effet, les auteurs de l'appel se déclarent «persuadés que tout consommateur conscient apprécie d'autant plus son achat lorsqu'il sait que l'article en question a été produit dans des conditions équitables et à des prix garantissant une rémunération décente au producteur» (2).

Les initiateurs estiment que les consommateurs éclairés peuvent contribuer à rendre les relations commerciales plus justes et plus équilibrées avec le Tiers Monde; d'autant plus qu'ils exercent une influence sur les distributeurs au moment où ils donnent leur préférence aux produits qui répondent aux exigences sociales et écologiques. "ll ne s'agit pas de prôner le commerce à tout prix, mais de favoriser à tous les niveaux le commerce équitable. Ceci pour ne pas devenir, contre notre gré, les profiteurs de salaires de misère ou les destructeurs de la nature dans les pays en voie de développement. Des produits socialement et écologiquement justes doivent trouver leur place sur le marché» (3).

La brochure explicative qui accompagne l'appel «Pour un commerce équitable avec le Tiers Monde» passe en revue un certain nombre de problèmes concrets que posent nos relations commerciales avec les PVD. Ainsi par exemple, elle parle des matières premières comme d'une urichesse qui crée la pauvreté», déplore les échanges modestes entre les pays du Sud, dénonce "le libre échange comme recette de développement» et remarque aussi que "le commerce avec le Tiers Monde participe en réalité à notre prospérité». Elle insiste enfin sur la nécessité d'informer le consommateur pour faire connaître les problèmes du développement et le sort des producteurs qui fournissent les produits qui se trouvent sur nos tables.

Du point de vue strictement économique les termes de cet appel et l'argumentation qui le soutient peuvent paraître déroutants. Mais réflexion faite, on peut constater qu'ils s'inscrivent dans le cadre d'un vaste débat entre diverses organisations de coopération au développement qui demandent l'élargissement du domaine économique par les considérations sociales, et les entreprises commerciales (souvent transnationales) des 
pays industrialisés qui estiment que les arguments socio-politiques ne devraient pas interférer sur l'action économique.

En tout état de cause, à la lecture de cet appel on peut se poser trois questions fondamentales:

- D'abord, il convient d'examiner si la rationalité économique est compatible avec le respect des valeurs sociales, voire même morales, que les initiateurs veulent introduire dans nos relations avec le Tiers Monde.

- En deuxième lieu, on peut se demander si l'application des principes de l'appel ne crée pas une "pression sociale» qui risque de compromettre la rentabilité économique des produits importés des PVD. Vu sous cet angle, il est à craindre qu'elle renforce, sans le vouloir, la vague de protectionnisme qui sévit déjà dans les pays industrialisés et qui frappe les produits en provenance des pays du Sud.

- Enfin, on peut se demander quels sont les pouvoirs dont disposent les consommateurs pour faire changer les pratiques actuelles des distributeurs en Suisse.

Pour examiner les enjeux de l'appel et en cherchant les réponses à ces questions, nous étudierons d'abord l'interpénétration des idées économiques et sociales dans le commerce international, pour envisager ensuite les tentatives des mouvements associatifs de dépasser l'appréciation purement économique des activités commerciales.

\section{Les vicissitudes du commerce avec le Tiers Monde}

La théorie du commerce international enseigne depuis deux siècles que les échanges internationaux sont mutuellement profitables pour les partenaires commerciaux. Cette idée constitue encore aujourd'hui le principe de base du GATT. Comme le remarque $M$. Arthur Dunkel, son directeur général, «le système des échanges multilatéraux repose sur l'idée généralement admise que le commerce international est ce que les Américains appellent 'un jeu à somme positive'. Le libre accès aux marchés mondiaux permet une certaine spécialisation et une efficacité certaine dans l'affectation des ressources dont tous les participants bénéficient quel que soit leur degré de développement» (4). En d'autres termes, le libre-échange, dont les fondements ont été élaborés par David Ricardo dans sa théorie des avantages comparatifs, aboutirait à une situation idéale, mutuellement profitable pour tous les Etats-nations.

Mais, si on l'examine de près, on peut constater que ce théorème, par l'utilisation que ses adeptes en font, est érigé en un jugement de valeur présenté sous forme d'une démonstration économique rigoureuse. Dans l'esprit normatif du libre-échange, le commerce mondial sans entraves est la seule voie qui aboutit au bien-être commun de l'humanité. Selon cette vision, l'échange international ne connaît ni perdants ni gagnants, il est utile à tous les participants, sans exception aucune, et les PVD ne constituent pas un cas particulier à cet égard (5). 
L'optique de l'Organisation de coopération et de développement économique (OCDE) est quelque peu plus nuancée. Elle reconnaît que les économies de ces pays sont "vulnérables» et que les deux mondes sont interdépendants. Or la notion de l'interdépendance entre le Sud et le Nord signifie non seulement la reconnaissance des «intérêts» mutuels, mais aussi elle débouche sur le principe de solidarité avec les partenaires qui sont plus faibles (6).

On peut donc remarquer à ce stade que la doctrine économique des relations commerciales internationales préconisée par le GATT et I'OCDE recèle bien quelques idées qui dépassent la sphère économique "pure" et laissent entrevoir en filigrane la présence de certaines valeurs sociales et morales.

Mais l'analyse des réalités du commerce international avec le Tiers Monde indique que les énoncés théoriques du libre-échange ne se vérifient pas dans son cas. Déjà en 1959, Myrdal a attiré l'attention sur le fait que cette doctrine n'arrive pas à uexpliquer pourquoi et comment les immenses inégalités économiques entre pays différents en sont venues à se produire et pourquoi elles ont tendance à s'aggraver" (7).

Les spécialistes de l'économie mondiale ont dû se rendre à l'évidence que la liberté du commerce ne peut être bénéfique que dans certaines conditions bien précises. Pour établir des relations commerciales équitables, chaque partenaire devrait être en mesure de s'appuyer sur des structures économiques internes suffisamment diversifiées afin de répartir les risques inhérents au commerce international sur plusieurs produits et sur différents marchés. Et précisément, les pays du Tiers Monde ne réunissent pas ces conditions, bien au contraire.

Dans la majorité des cas, les PVD sont fournisseurs de matières premières et vivent souvent de l'exportation d'un seul produit. Or, les structures de production et l'organisation des marchés des produits de base sont défavorables aux pays pauvres. Comme leurs débouchés se trouvent uniquement dans les pays industrialisés et qu'ils ne disposent pas de marché interne pour les produits qu'ils exportent, leur dépendance est accrue vis-àvis de la demande extérieure dont l'instabilité se reflète dans les fluctuations des prix mondiaux.

D'autres facteurs bien connus des économistes contribuent aussi à l'inégalité du commerce avec le Tiers Monde (par exemple la substitution des produits de base naturels, comme le coton ou le caoutchouc, dès que le niveau de prix est élevé; la difficulté d'adaptation aux changements du marché ; la faible élasticité du revenu, etc.). En même temps, dépendants des exportations qui leur procurent des revenus indispensables, les pays pauvres sont souvent contraints de vendre leurs produits, même dans les conditions qui leur sont défavorables sur les marchés internationaux (par exemple, baisse des prix). On peut dire que si l'échange est effectivement profitable pour quelqu'un, c'est pour les partenaires commerciaux des PVD, car il est sans réciprocité. Ainsi, le rôle du fournisseur des matières premières pour les pays industrialisés représente plus un «désavantage com- 
paratif» que le contraire, chanté par les épigones de Ricardo.

Dans les conditions actuelles, le commerce international draine les ressources du Tiers Monde au lieu de l'enrichir. Comme le remarque une étude de la CNUCED au sujet du commerce international de la banane: "La chute des prix des bananes a profité aux consommateurs des pays développés et a privé les pays exportateurs des avantages résultant des innovations... Ainsi, pendant des décennies, il y a eu un transfert net de ressources réelles en faveur des pays développés à partir des pays en voie de développement" (8).

C'est dans le cadre de la Conférence des Nations Unies sur le commerce et le développement (CNUCED) que s'élabore une autre vision des problèmes commerciaux avec le Tiers Monde. Dès 1964, lors de sa première réunion, la CNUCED déclara que le commerce international devait être régi par «des principes compatibles avec les réalisations du progrès économique et social» (9).

Les différents documents de la CNUCED présentent une analyse nouvelle des effets du commerce international pour les PVD. Ces études mettent en cause les inégalités des partenaires qui entraînent l'inéquité des échanges: "Il est maintenant reconnu que l'expansion économique était fondée jusqu'à présent sur un gaspillage des ressources mondiales et sur une mauvaise répartition de la consommation et l'on se rend compte désormais de la nécessité d'une répartition plus équitable du revenu global» (10). En fait, avec l'avancement des travaux au sein de la CNUCED, on peut observer la rupture avec la vision idéaliste du commerce international basée sur le libre-échange. Dès lors, on doit aménager les structures qui ne donnent pas satisfaction et établir les bases des solutions nouvelles. La «diplomatie de développement", ébauchée par la CNUCED, cherche un règlement négocié pour amener les partenaires à créer un ordre économique mondial global qui corrige les mécanismes des marchés en faveur des désavantagés.

Les idées en matière de commerce international évoluent vers les conceptions qui englobent de plus en plus les critères qui ne sont pas nécessairement économiques. L'interdépendance et la restructuration négociée restent les maîtres-mots des relationss économiques internationales aujourd'hui, même si certains politiciens semblent redécouvrir (en paroles) les vertus de l'économie de marché.

\section{Les produits peuvent-ils être «appropriés»?}

A l'échelle des activités quotidiennes, le commerce ne se réduit pas à une fonction purement économique, mais s'inscrit dans un contexte socio-culturel. Le marché s'organise autour de la rencontre entre acheteurs et vendeurs et l'échange se déroule selon les règles et rites qui varient selon les cultures. On peut évoquer à cet égard les scènes de marchandage sur les marchés partout dans le monde. 
Avec l'avènement des supermarchés, la dimension sociale de l'acte d'achat s'est transformée de fond en comble. Le contact personnel entre producteur et acheteur a été remplacé par les moyens de communication publicitaires et par la «mise en scène» de la marchandise aux points de vente.

Dans une société de consommation, le produit (objet/marchandise) occupe un rôle central: il doit "se vanter», donc se vendre à travers son image. Il ne se définit plus uniquement par sa qualité utilitaire, ou par I'usage que peut en faire l'acheteur, mais par son effet psychologique. Comme le remarque Baudrillard, «l'objet n'a plus de fonction, il a une vertu: c'est un signe» (11). Autrement dit, le choix des consommateurs se fait de plus en plus en tenant compte des caractéristiques immatérielles et subjectives incorporées dans les produits. C'est ainsi que les "satisfactions" imaginaires qu'apportent les marchandises appartiennent à la sphère des valeurs sociales individuelles ou collectives.

On peut constater que les entreprises modernes ont tôt reconnu que dans une société de consommation, l'achat est un phénomène social. Depuis lors, par le biais du "marketing», elles mettent l'accent sur les critères psychologiques et manipulent en même temps les codes, les signes et les symboles sociaux pour faire acheter leur marque. La mise en œuvre des méthodes de marketing permet aux entreprises de modifier constamment la forme et l'image des produits, d'utiliser les techniques de communication de masse (publicité et promotion) et de mieux gérer la diffusion et la distribution de leurs produits. En effet, le marketing est devenu une des fonctions fondamentales des entreprises pour attirer les consommateurs et pour contrôler les marchés.

Il est utile donc de rappeler que les milieux économiques et les dirigeants des entreprises ont largement contribué à introduire les considérations sociales dans le domaine économique. Or, dans une société où les produits véhiculent un message social, il est inévitable que certains groupes remettent en question les valeurs que ces produits renferment et transmettent.

Mais la critique des objets dans la socièté de consommation ne s'arrête pas au niveau de l'étude psycho-sociologique. Pour saisir les réalités de la consommation, elle veut d'abord connaître les conditions réelles de la production des marchandises. Le premier réflexe des consommateurs conscients envers les produits nocifs pour la santé est de remonter la filière de la production pour savoir ce que contiennent les produits. Le revirement se produit par le retour aux sources: pour eux, le contenu des marchandises prime la forme. La prise de conscience qu'il existe des producteurs derrière les produits ne vient à l'esprit des consommateurs que plus tard.

Pour examiner les problèmes que posent les produits en provenance des PVD, c'est I'Organisation internationale des Unions de consommateurs (OIUC) qui a offert une plate-forme pour la rencontre entre les consommateurs des pays industrialisés et les producteurs du Tiers Monde. L'OIUC s'est préoccupée d'abord de l'utilisation des pesticides et herbicides dange- 
reux pour la santé des acheteurs (sous-entendu dans les pays industrialisés); mais elle a débordé très vite sur d'autres questions touchant aux conditions de vie et de travail des paysans du Tiers Monde.

Lors du sixième congrès de l'OIUC en 1981, on a décidé de créer un réseau d'échange d'informations sur les produits dangereux. C'est à cette occasion que les associations de consommateurs ont étudié la possibilité de la prise en compte de facteurs sociaux et économiques lors de l'achat des produits en provenance des PVD. Les groupements des pays industrialisés ont décidé de rendre leurs adhérents plus conscients de la manière dont leur mode de vie et la politique commerciale de leurs pays affectent d'autres pays (12).

Ainsi esî née la notion du "produit approprié», à l'instar des "technologies appropriées". II s'agit de termes jumeaux: un produit approprié est le résultat de l'application des techniques qui le sont aussi. Autrement dit, ces produits devraient être fabriqués dans des conditions sociales et écologiques adaptées aux besoins de la population locale, au lieu de produire uniquement en fonction des marchés extérieurs à l'aide de techniques importées. Cette conception intègre les activités productrices dans un contexte social et écologique plus large.

Par exemple, la fabrication des produits appropriés se fait à petite échelle, avec peu ou pas de capital extérieur, en utilisant les technologies et les ressources indigènes. En outre, la production "appropriée» vise à créer des emplois et à maintenir les places de travail et respecte les équilibres écologiques.

Le concept des activités économiques "appropriées» (technologies et produits) qui tiennent compte des critères écologiques et sociaux de la production devrait déboucher logiquement sur l'idée de la consommation «appropriée». II interpelle l'acheteur et pose le problème de sa responsabilité au moment de l'achat et de la consommation. En fait, on peut constater que la responsabilité des consommateurs s'établit sur trois niveaux: d'abord, vis-à-vis de lui-même, pour sauvegarder sa santé, deuxièmement, à l'égard des producteurs d'ici et du Tiers Monde et enfin, sa responsabilité est aussi engagée envers l'environnement.

\section{Le mouvement des consommateurs}

Parmi les différents mouvements associatifs, le mouvement des consommateurs est celui qui exprime en premier lieu le souci d'introduire la justice et l'équité dans les relations commerciales. En fait, la défense des intérêts des consommateurs est inséparable des objectifs sociétaux plus larges.

Alors que la fin du XIXe siècle était marquée par la création des syndicats ouvriers, la deuxième partie du XXe siècle a vu apparaître le mouvement de défense d'un groupe social diffus, celui des consommateurs. Avant d'être militants, organisés en mouvement, ce sont les acheteurs isolés qui ont exprimé de façon sporadique leur mécontentement devant les abus commerciaux et devant les méthodes de marketing des entreprises. La 
création des associations de consommateurs est le résultat du regroupement des acteurs socio-économiques dispersés, laissés pour compte par les organisations politiques (partis) ou sociales (syndicats et associations professionnelles). Ce mouvement associatif est donc né de la conviction que seule une force sociale organisée peut se constituer en interlocuteur reconnu et respecté face aux corporations économiques puissantes (fabricants et distributeurs, dont certains transnationaux).

Au cours des années, les revendications des consommateurs sont allées du particulier au général. La Fédération romande des consommatrices (FRC), par exemple, en partant de quelques réclamations au sujet des problèmes mineurs (des «broutilles»), a découvert peu à peu les failles dans l'organisation économique et sociale: les lacunes dans la législation, les produits dangereux pour la santé ou l'aberration de l'organisation agricole. «ll y a eu un continuel élargissement», écrit la première présidente de la FRC dans une brochure éditée à l'occasion du vingtième anniversaire de l'organisation (13).

La protection des consommateurs est devenue une action sociale permanente. Mais paradoxalement, c'est sur ce plan que se situent les limites de son engagement en faveur des causes qui ne touchent pas directement aux intérêts de ses membres. Les organisations de consommateurs agissent avant tout sur le terrain pour améliorer les pratiques commerciales des fabricants et des distributeurs. Ainsi, même si les relations commerciales avec le Tiers Monde préoccupent la FRC, ce n'est pas elle qui se trouvera en première ligne. Par contre, l'action des autres groupements en faveur des PVD peut compter sur l'appui de la FRC. II se manifeste en particulier par la publication d'articles bien documentés dans le journal de la FRC, "J'achète mieux». Ces articles sur les problèmes touchant aux produits importés du Tiers Monde informent les lecteurs et les font réfléchir.

Pour illustrer l'attitude de l'organisation des consommateurs à l'égard du commerce avec les PVD, on peut citer un article paru dans le centième numéro de son journal, intitulé: "Profiter?». "Plusieurs fois, nous avons attiré votre attention sur notre choix de consommateurs et ses conséquences pour les PVD. L'exemple récent du prix du café nous incite à y revenir... Les consommateurs suisses se trouvent devant un choix: ils peuvent soit profiter de la baisse actuelle des cours mondiaux et trouver du café extrêmement avantageux, soit donner la préférence au café du Nicaragua, vendu par les Magasins du Monde et accepter de ce fait de payer le café à un prix plus juste pour les producteurs du Tiers Monde. Ce choix de principe a plus d'importance qu'il n'en a l'air. Nous sommes en effet habitués à considérer que tout nous est dû et que les baisses de prix sont bonnes à saisir. II faut essayer de voir un peu plus loin, les conséquences pour les autres des rabais qu'on fait miroiter sous nos yeux. Devons-nous profiter des prix dérisoires de nombreux produits du Tiers Monde obtenus grâce à l'exploitation des plus faibles ou tenter chaque fois que c'est possible de corriger cette situation? Pour le café nous avons de la chance, puisque nous avons le choix» (14). 


\section{La critique sociale du commerce}

La défense des consommateurs fait partie du militantisme socio-économique au même titre que les causes défendues par les écologistes ou tiersmondistes. De nombreuses préoccupations économiques et sociales sont communes aux diverses associations, comme la pollution, le gaspillage des matières premières, etc. Mais, même si les mouvements féministes, écologistes ou tiers-mondistes se mettent d'accord sur les mêmes idées de changement de société, chacun interviendra dans son domaine, avec son style particulier. La période des grandes mobilisations semble être révolue, elle a laissé place à l'action quotidienne menée par les organisations plus ou moins structurées, à qui le pouvoir public a aménagé des espaces de consultation au sein des institutions.

Dans ces conditions, il n'est pas étonnant de constater que les revendications en faveur du Tiers Monde ont été reprises par les organisations d'entraide qui n'ont pas d'intérêts particuliers à défendre. En effet, travailler pour la cause du Tiers Monde, dans un pays dit développé, suppose une certaine dose d'idéalisme et d'abnégation. Cette attitude apparemment altruiste s'explique cependant aisément par un changement de vision pour appréhender les différents problèmes de notre temps. Les animateurs de ces groupes optent pour une perspective globale et à long terme, dans laquelle les intérêts particuliers immédiats s'effacent.

Plusieurs étapes ont été franchies par les organisations tiers-mondistes ou œuvres d'entraide qui souhaitent rendre le commerce plus équitable avec le Tiers Monde. Nous en envisageons trois exemples qui montrent le cheminement de l'idée qui veut transformer les relations commerciales entre la Suisse et les PVD. En effet, aux yeux des tenants de cette pensée, le commerce plus juste est la condition primordiale du développement et de l'émancipation des producteurs du Tiers Monde.

\section{a) Les Magasins du Monde}

Le premier pas concret dans la direction de revoir les liens commerciaux entre la Suisse et le Tiers Monde a été fait par les Magasins du Monde.

L'Association romande des Magasins du Monde était fondée en juillet 1974, suite à une action de vente de café soluble de Tanzanie. Dix ans plus tard, il existe environ cinquante points de vente, soutenus par une trentaine d'associations. Les fondateurs des Magasins du Monde poursuivent deux buts: ils veulent accorder une meilleure rémunération aux producteurs du Tiers Monde et ils souhaitent informer les acheteurs des pays industrialisés sur les problèmes des pays en voie de développement.

En ce qui concerne le premier objectif, ils s'approvisionnent directement chez les petits producteurs, en coupant court aux circuits commerciaux habituels. Ils souhaitent partager ainsi plus équitablement les gains réalisés par le commerce. Comme ses fondateurs l'expliquent : «Nous tendons à un partage plus juste des gains et des richesses et nous considérons le plus pauvre comme notre partenaire» (15). En même temps, les Magasins du Monde utilisent les produits et leurs emballages comme des supports pour 
transmettre des informations sur le pays producteur concerné. Chaque produit est vendu avec une feuille explicative pour amener les acheteurs à réfléchir sur les conditions de vie et de travail des paysans du Tiers Monde au moment où ils consomment le produit.

Cette tentative est certes limitèe, mais durable, car elle survit depuis une quinzaine d'années en marge du système commercial dominé par les grands distributeurs (Migros et Coop). Elle a valeur d'exemple pour démontrer qu'il peut exister des alternatives commerciales opérantes.

\section{b) M-Renouveau}

En deuxième lieu, il est intéressant d'évoquer les principes élaborés par le mouvement $\mathrm{M}$-Renouveau au sujet d'une autre forme de commerce. II regroupe, dès 1979, les coopérants dissidents qui ont projeté de rénover la politique commerciale de la Migros, le plus grand distributeur en Suisse.

La Migros est un phénomène commercial unique. Son organisation coopérative, ses résultats (7,5 milliards de francs en 1979, trois milliards de plus en 1985), sa gestion exemplaire, son poids dans la vie politique et culturelle font de la Migros une des plus grandes puissances économiques de la Suisse. En effet, au cours de quarante années de son existence, Migros est devenue le plus gros acheteur des produits agricoles et le plus important distributeur des produits alimentaires. Elle est un des plus grands organisateurs de voyages et dirige le plus important organisme de formation des adultes. Elle est aussi présente dans les secteurs d'assurance et de banque ; elle possède sa propre presse dont le tirage est un des plus importants de Suisse.

Et précisément, au zénith de sa réussite, un groupe de personnes venant d'horizons divers (tiers-mondistes, consommateurs, politiciens) a créé un courant de contestation sous forme de M-Renouveau pour demander le rétablissement de la démocratie coopérative et le changement des méthodes commerciales appliquées par Migros.

Le programme de $\mathrm{M}$-Renouveau englobe parmi d'autres, le respect de l'écologie, le soutien d'une politique favorable au Tiers Monde, la suppression de méthodes dévoreuses d'énergie et polluant l'environnement, de même que I'humanisation du travail.

En ce qui concerne le Tiers Monde, le texte élaboré par M-Renouveau garde toute sa fraîcheur et actualité encore aujourd'hui :

- «M-Renouveau exige que la Migros mène une politique responsable en matière de développement. En offrant toujours plus de produits venant des pays du Tiers Monde, Migros influence la situation de milliers de travailleurs vivant dans ces pays. II faut que Migros soit bien consciente de telles implications, les mettre en évidence aux yeux des consommateurs et constitue un exemple pour les autres entreprises».

- «Nous préconisons de renoncer à des produits qui renforcent la position des firmes multinationales dans les pays en développement et qui renforcent la monoculture. Nous allons chercher et encourager de nouveaux partenaires commerciaux qui produisent en petites unités et 
s'organisent sur une base coopérative ou d'aide mutuelle.»

$M$-Renouveau voulait également mobiliser les consommateurs et les rendre plus conscients des conséquences sociales de leurs achats:

- «Par nos achats à la Migros, nous prenons part au marché mondial qui porte préjudice aux pauvres. Or, sans nous culpabiliser, nous pouvons consommer en ayant davantage conscience des problèmes. Nous pouvons aussi infléchir la politique de la Migros pour l'amélioration des conditions d'achat et d'échange ; des mesures concrètes peuvent constituer une ouverture pour un commerce plus égalitaire» (16).

Le programme de $M$-Renouveau, véritable creuset d'idées nouvelles, inhabituelles et choquantes à l'époque (pourtant pas si lointaine) a suscité aussitôt une levée de boucliers des milieux économiques qui, d'une manière générale et en temps normal, ne portent pas toujours Migros dans leur cœur. Quant au principal intéressé, la Migros, elle a mis en œuvre des moyens de propagande non négligeables (avant tout son journal) pour contrer ses détracteurs.

L'argument contre les revendications de M-Renouveau est simple: on oppose l'efficacité économique aux rêves idéologiques. Un des représentants d'une organisation patronale a déclaré que l'approche de M-Renouveau usemble faire bon marché de l'intérêt même des consommateurs et du grand public en général. Pénalisons Migros par toutes sortes d'obligations, louables individuellement, mais insupportables commercialement. Car on ne s'improvise pas spécialiste du commerce de détail, technicien en usines de production, manager professionnel» (17).

Après les élections des organes directeurs de Migros où le groupe n'a recueilli que $20 \%$ des voix, M-Renouveau s'était mis en veilleuse. D'autres ont repris le flambeau de la cause du commerce plus juste avec le Tiers Monde.

\section{c) La clause sociale du contrat Migros-Del Monte}

En 1981, la Déclaration de Berne a lancé une campagne pour dénoncer les problèmes de la famine dans le Tiers Monde («la faim est un scandale»). Elle a eu pour but de sensibiliser le consommateur aux conditions déplorables de production de l'ananas en conserve, fabriqué aux Philippines par Del Monte, une des plus importantes sociétés agro-alimentaires transnationales.

Sous la pression de cette action (20.000 personnes ont signé l'appel), en 1983, Migros et Del Monte ont signé une clause sociale. Elle stipule que «le fournisseur se porte garant, face à la Migros, de mettre à la disposition des travailleurs des méthodes de production supérieures à la moyenne, tant du point de vue social qu'économique" (18).

La signature d'une clause sociale qui engage la responsabilité des fournisseurs de mettre en place des conditions de travail décentes, est un acquis social important en faveur des producteurs qui exportent leurs produits dans les pays industrialisés. Elle est le résultat concret d'un long débat (sans dialogue) entre les groupes militant en faveur du Tiers Monde et les 
entreprises. Certes, les représentants des organisations d'entraide ne sont pas entièrement satisfaits, ils auraient souhaité que la clause sociale soit formulée d'une manière plus précise et qu'elle tienne compte des normes établies par les conventions de l'Organisation internationale du travail.

Mais la mise en place d'une clause sociale démontre qu'il est possible de modifier les conditions de production dans les PVD par un accord entre fournisseur étranger et distributeur suisse, sous la pression des organisations qui mobilisent les consommateurs. Ce fait est non seulement encourageant pour l'action des mouvements tiers-mondistes, mais encore il "ouvre un nouveau champ d'information, de campagne et de pression au service d'une réelle politique de développement», constate la Déclaration de Berne. L'appel «Pour un commerce équitable avec le Tiers Monde» s'inscrit dans cette lignée.

\section{La banane: symbole du commerce inéquitable}

Le commerce de la banane est la meilleure illustration de l'échange inéquitable entre exportateurs du Tiers Monde et importateurs des pays industrialisés. Produite dans des conditions sociales précaires, acheminée vers les consommateurs par les entreprises transnationales qui dominent les marchès internationaux, la banane est un des derniers produits où survivent encore les rapports coloniaux. Les relations entre les «républiques bananières» d'Amérique latine et les firmes américaines transnationales qui défendent avec acharnement leur pouvoir, transforme le monde des bananes en un véritable microcosme agro-politique où l'on retrouve toutes les méthodes de pression et de violence dignes des romans à sensation. II convient donc de passer en revue la structure actuelle du commerce international de la banane dont les enjeux économiques et sociaux dépassent le cadre étroit du produit.

La banane est un fruit universel: consommée sous diverses formes (légume, fruit, bière), elle constitue l'alimentation de base de la population dans un certain nombre de pays tropicaux. En même temps, exportée, elle est le fruit le plus répandu en Europe et aux Etats-Unis. Grâce à la distribution de masse, cette denrée - qui était encore il y a peu de temps un produit de luxe - est devenue aujourd'hui un fruit banal et bon marché sous toutes les latitudes.

L'exploitation commerciale de la banane remonte à la fin du XIXe siècle en Amérique latine et le développement des marchés internationaux de ce produit est étroitement lié à l'expansion des grandes entreprises transnationales bananières, notamment à celle de United Brands (anciennement United Fruits qui commercialise les bananes sous la marque Chiquita), Del Monte (filiale du groupe R.J. Reynolds) et la Standard Fruit (achetée par Castle \& Cooke en 1964).

Environ $40 \%$ de la production mondiale de la banane sont exportés, soit en moyenne 6 millions de tonnes par an. L'exportation des bananes est devenue une source de revenus importants pour plusieurs pays d'Amérique 
latine et centrale. Ainsi, par exemple, la part des bananes dans les exportations totales représente $29 \%$ pour le Costa Rica, $41 \%$ pour l'Equateur, $48 \%$ pour le Honduras et $58 \%$ pour le Panama. Depuis plusieurs années le marché mondial de la banane est caractérisé par une surproduction qui a provoqué l'effondrement des prix. Cette tendance défavorable explique la stratégie de certaines compagnies bananières transnationales (notamment de United Brands) qui se détournent peu à peu de l'exploitation des plantations de bananes dans les pays en voie de développement et s'orientent vers la production d'autres produits tropicaux plus rentables, comme l'huile de palme.

Dans sa forme commerciale, destinée à l'exportation, la banane est vendue uniquement comme un fruit frais, car elle ne peut être ni surgelée, ni conservée en boîte. Etant donné qu'il s'agit d'une denrée périssable, le transport des bananes (généralement par la voie maritime) exige des soins et techniques particuliers (bateaux frigorifiques). Dans les pays de consommation, le fruit est stocké chez les mûrisseurs avant la distribution chez les détaillants. En fait, les trois entreprises transnationales contrôlent toute la filière qui mène de la production à la consommation des bananes.

Pour contrebalancer la place prépondérante des entreprises transnationales qui gardent leur situation dominante dans la production, le transport et la commercialisation des bananes, les pays producteurs ont créé I'Union des pays exportateurs de banane (UPEB). Mais I'UPEB n'a pu acquérir, au cours des années, qu'environ $37 \%$ du marché mondial de la banane, contre $63 \%$ qui sont toujours acquis aux compagnies transnationales. Ces dernières s'opposent à toute tentative de créer des marchés qui pourraient échapper à leur contrôle.

La meilleure illustration en est la "guerre des bananes» entre les firmes transnationales et les pays producteurs qui a éclaté en mars 1974 par l'accord conclu entre le Guatémala, le Costa-Rica, le Honduras et le Panama, pour instituer une taxe d'exportation de 1.-US\$ par carton de bananes. Cette tentative des producteurs de s'organiser et de tirer un meilleur revenu de leurs exportations de bananes s'est heurtée à l'opposition violente des compagnies bananières qui ont vu leur monopole menacé. Ces dernières n'ont pas hésité à recourir aux menaces, à l'intimidation, au refus d'achat ou aux pots-de-vin pour empêcher l'application de la taxe sur les bananes. Le conflit autour de la taxe à l'exportation entre les membres de I'UPEB et les firmes transnationales subsiste toujours, d'autant plus que la saturation du marché des bananes exerce une pression supplémentaire sur les pays producteurs. Par exemple, en 1984, sous la menace de fermer ses installations au Panama, United Brands est parvenue à imposer une baisse de la taxe de $\$ 0.60$ à 0.42. Par contre, elle s'est retirée du Costa Rica qui a maintenu une taxe de $\$ 0.70$, malgré les menaces. On ne peut que constater que le pouvoir des transnationales bananières est pratiquement intact encore aujourd'hui, de même que «l'économie mondiale de la banane reste une économie coloniale" (19). 
L'inégalité de l'échange entre producteurs des pays en voie de développement et consommateurs des pays riches se manifeste aussi dans la formation du prix de la banane. Si l'on décompose le prix de vente d'un kilo de bananes dans un magasin en Suisse, on peut remarquer que la part des pays exportateurs ne représente qu'environ $20 \%$ du prix de vente au détail (et celle des paysans producteurs ne s'élève qu'à environ $11 \%$ ), le reste englobe les coûts des services comme le transport, le mûrissage et les marges commerciales des divers distributeurs. Ainsi, le rapport entre le prix à la production et le prix payé par le consommateur varie entre 4,5 et 5,0. Autrement dit, l'acheteur débourse quatre et demi à cinq fois plus que n'obtient le producteur.

Ce calcul montre la répartition inégale du revenu entre différents agents économiques qui interviennent dans la production et la distribution de la banane. II permet aussi d'établir qu'une des caractéristiques fondamentales de la société de consommation est le fait que le travail du producteur des matières premières est peu rémunéré. Par contre, la valeur ajoutée des marchandises augmente au fur et à mesure que l'on remonte dans la filière de la distribution. L'ensemble des actitivés commerciales qui contribuent à l'acheminement des produits vers les acheteurs sont en réalité les plus rémunératrices.

Le constat de la domination des entreprises transnationales dans les pays producteurs et de la (dé)formation des prix de la banane a amené un groupe de femmes de Frauenfeld («Bananenfrauen») à se pencher, dès 1974, sur les problèmes que pose la commercialisation des bananes pour les consommateurs suisses. Partant de l'idée que la banane étant bon marché - meilleur marché même que les pommes suisses - le consommateur l'achète sans réfléchir, en profitant des prix bas. Or, la pauvreté du Tiers Monde, l'effondrement des prix des bananes et les activités des entreprises transnationales devraient susciter une réflexion sur les produits achetés dans les PVD.

Le groupe d'action a proposé alors de majorer de 15 centimes le prix de vente du kilo de bananes et de verser les sommes récoltées aux organisations d'entraide pour financer les projets de développement dans les pays producteurs de bananes. Un "label» était créé pour marquer les bananes vendues dans le commerce avec ce supplément de prix. Par ailleurs, le groupe a tenté d'informer le grand public sur la situation des producteurs de bananes. En dix ans, malgré le refus de collaboration des grands distributeurs comme Migros, I'action des «Bananenfrauen» a permis de recueillir 100.000 francs. Cette somme a èté versée à un projet de développement au Honduras.

La campagne "Action Banane Nica», en faveur de l'introduction des bananes du Nicaragua en Suisse, peut être considérée en fait comme une manifestation de solidarité concrète dans le domaine du commerce avec le Tiers Monde (20). Comme il s'agit de la vente des bananes, produit symbolique, elle s'appuie donc en partie sur l'action modeste, mais tenace, de la sensibilisation des acheteurs entreprise par les femmes de Frauenfeld. Le 
succès de la campagne montre en tout état de cause que le public devient de plus en plus réceptif aux problèmes économiques et sociaux des populations du Tiers Monde.

Mais il est trop tôt pour crier victoire. Une fois de plus la réalisation du projet de commercialiser un produit du Tiers Monde avec les arguments de solidarité s'est heurtée à la résistance des distributeurs en place, avant celle de Migros. Par contre, Coop-Suisse, la deuxième chaîne de distribution, a laissé la liberté aux coopératives régionales de vendre ou non les bananes du Nicaragua. Le refus de Migros est motivé par l'importance qu'elle attache à la qualité du produit. M. Eric Gugelmann, chef du département marketing de Migros, a déclaré: «Par principe, nous sommes ouverts à tous ceux qui désirent vendre, à condition que le prix et la qualité soient corrects. Dans le cas du Nicaragua, nous nous tenons clairement en dehors du problème politique qui entoure ces bananes, car nous voulons faire du commerce, non de la politiquen (21).

En principe, la Migros n'achète pas de bananes qui transitent par le canal de Panama, car le trajet parcouru devient trop long et les bananes perdent leur "aspect» qui les fait vendre. Car, les études de marché indiquent que les consommateurs achètent les bananes «avec les yeux»: ce ne sont pas les qualités gustatives qui sont les plus importantes dans le choix des bananes, mais l'apparence extérieure du fruit.

L'opération "Action Banane Nica» a apporté l'enseignement aux initiateurs que, pour obtenir un succès durable dans le commerce, ils doivent se préoccuper aussi des problèmes techniques de la commercialisation. La bonne volonté et les idées (si justes soient-elles) ne remplacent pas la gestion et le savoir-faire (au contraire, elles les renforcent). Dans le commerce international en général, et dans celui de la banane en particulier, on doit observer un certain nombre de règles afin que la marchandise soit effectivement vendable: mode de transport et équipement portuaire appropriés, durée de séjour dans les cales des navires, conditions de stockage et recherche de points de vente. En fait, le marché est un tissu de relations entre une série d'acteurs (transporteurs, négociants, distributeurs) en plus des producteurs et des acheteurs qu'il faut connaître ou créer, si l'on veut réussir.

Quelle conclusion peut-on tirer de ce débat d'idées qui débouche de plus en plus sur l'action concrète sur le terrain commercial? II semble qu'actuellement on se trouve à mi-chemin. L'acheteur devient certainement plus conscient des conséquences sociales de son achat, mais les structures du commerce en Suisse ne se sont pas transformées pour autant. En tout cas, le mouvement de solidarité avec les producteurs du Tiers Monde ne peut être considéré comme le fait d'une minorité d'idéalistes, mais il représente un des courants de la pensée contemporaine qui cherche à réintégrer la dimension sociale aux activités économiques. 


\section{NOTES ET REFERENCES BIBLIOGRAPHIQUES}

1. Il s'agit d'une action commune des organisations suivantes:

Action de Carême, Caritas, EPER (Entraide protestante suisse), Helvetas, Oeuvre suisse d'entraide ouvrière (OSER), Pain Pour le prochain et Swissaid.

2. Caritas Informations, $1 / 86$, p. 1.

3. Pour un commerce équitable avec le Tiers Monde. Brochure éditée par l'Action pour un commerce équitable avec le Tiers Monde, Lausanne, s.d., p. 5.

4. «Commerce international et croissance économique». Discours d'Arthur Dunkel devant le European Atlantic Group. GATT/1355, le 20 février 1984.

5. GATT. Politique commerciale et prospérité. Des propositions d'action. (Rapport de sept personnalités éminentes.) Genève, GATT, 1985, $67 \mathrm{p}$.

6. OCDE. Interdépendance économique mondiale et évolution des relations NordSud. Paris, OCDE, 1983, p. 35.

7. MYRDAL, Gunnar. Théorie économique et pays sous-développés. Paris, Présence Africaine, 1959, p. 173.

8. CNUCED. Le système de commercialisation et de distribution de la banane. Rapport du Secrétariat de la CNUCED, 1974. TD/B/C.1/162, p. 94.

9. NATIONS UNIES. Les actes de la première CNUCED. New York, ONU, 1965, vol. I, «Principes généraux et particuliers», pp. 20-27.

10. CNUCED. Nouvelles orientations politiques internationales en matière de commerce et de développement. New York, Nations Unies, 1976, TD/B/530/Rev.1, p. 3.

11. BAUDRILLARD, Jean. Le système des objets. La consommation des signes. Paris, Denoël/Gonthier, 1975, p. 100. Voir aussi, LEPAGE, Henri, Vive le commerce. Paris, Dunod, 1982, $153 \mathrm{p}$.

12. WEISS, Dimitri et CHIROUZE, Yves. Le consumérisme. Paris, Sirey, 1984, 387 p.

13. SCHMITT, Ariane. La FRC, un partenaire majeur, 1959-1979. Genève, FRC, s.d., p. 47.

14. J'achète mieux, no 100,1982, p. 19.

15. MORSIER, Françoise de. "Promotion commerciale de produits artisanaux en jute du Bangladesh». Annuaire Suisse-Tiers Monde, Genève, IUED, no 2, 1982, pp. 169-175.

16 RENOUVEAU, mensuel édité par I'Association M-Renouveau, les 19 février et 11 mars 1980.

17. SUNIER, JEAN. "M-Renouveau saurait-il mieux diriger la Migros?» Journal de Genève, le 21 avril 1980.

18. "Philippines. Clause sociale entre Migros et Del Monte.» Vers un développement SOLIDAIRE, no 73, juillet 1984, pp. 17-18.

19. CHALMIN, Philippe et GOMBEAUD, Jean-Louis. Les marchés mondiaux en 198485. Economica, Paris, 1985, p. 100.

20. En effet, suite à la décision des Etats-Unis de suspendre leur commerce avec le Nicaragua en mai 1985, ce pays a perdu d'un jour à l'autre son marché d'exportation pour sa banane qui se situe exclusivement en Califormie. Les quantités des bananes exportées par le Nicaragua s'élèvent annuellement à 5 millions de cartons (90.000 tonnes). Ce refus d'achat représente une perte de 30 millions de dollars, soit environ le dixième du total des recettes d'exportations du Nicaragua.

21. "Migros et les bananes», interview avec M. Erich Gugelmann, vice-président de la délégation de l'administration de la FCM et chef du département Marketing, CONSTRUIRE, 2 avril 1986,p. 6. On peut encore remarquer que le marché suisse des bananes absorbe annuellement 60.500 tonnes, dont $45 \%$ sont vendues par 
Migros. A titre de comparaison, Migros seule écoule sur le marché une quantité correspondante à deux tiers des exportations totales des bananes du Nicaragua. Le tableau suivant indique les principales sources d'importation des bananes de la Suisse.

\section{Importations des bananes en 1985}

\section{Pays}

Volume

Valeur

en $1.000 t$

en mio Fr

Panama

26,6

35,2

Honduras

17,8

24,0

Costa Rica

11,9

16,7

Colombie

2,1

RFA

Divers

1,1

2,7

1,0

1,5

1,8

Total 1985

60,5

81,9

Prix moyen $/ \mathrm{kg}$

Fr 1,35

Total 1984

59,2

73,1

Prix moyen $/ k g$

Fr 1,23

Source: Statistiques douanières, 1985 